\title{
Nature of a white opaque substance visualized by magnifying endoscopy in colorectal hyperplastic polyps*
}

\section{다 (i)}

\author{
Authors \\ Haraoka $^{3}$, Akinori Iwashita ${ }^{3}$ \\ Institutions \\ 1 Department of Endoscopy, Fukuoka University Chikushi \\ Hospital, Fukuoka, Japan \\ 2 Department of Gastroenterology, Fukuoka University \\ Chikushi Hospital, Fukuoka, Japan \\ 3 Department of Pathology, Fukuoka University Chikushi \\ Hospital, Fukuoka, Japan
}

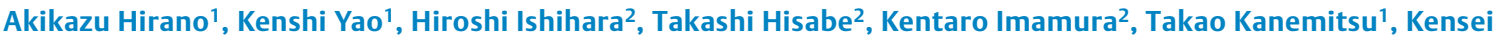

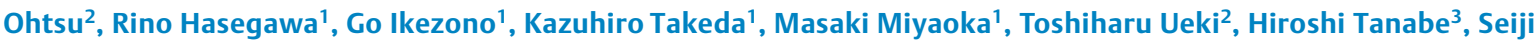

submitted 17.11 .2020

accepted after revision 23.2.2021

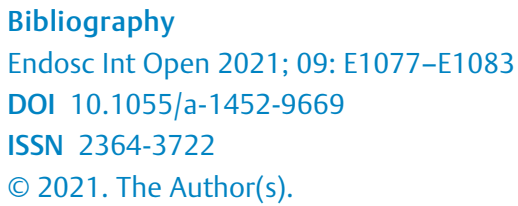
Commons Attribution-NonDerivative-NonCommercial License, permitting copying and reproduction so long as the original work is given appropriate credit. Contents may not be used for commercial purposes, or adapted, remixed, transformed or built upon. (https://creativecommons.org/licenses/by-nc-nd/4.0/)

Georg Thieme Verlag KG, Rüdigerstraße 14,

70469 Stuttgart, Germany

Corresponding author

Kenshi Yao, MD, PhD, Department of Endoscopy, Fukuoka University Chikushi Hospital, Zokumyoin, Chikushino City, Fukuoka 818-8502, Japan

Fax: +81-092-929-2630

yao@fukuoka-u.ac.jp

\section{ABSTRACT}

Background and study aims A white opaque substance (WOS) has been observed in the epithelia of gastric, duodenal, and colorectal epithelial adenomas and carcinomas, using magnifying endoscopy (ME). The WOS has been reported to be derived from a dense accumulation of minute lipid droplets in the epithelium. This study aimed to investigate whether the WOS in colorectal hyperplastic polyps was derived from lipid droplets accumulated in the epithelium, as observed in the case of gastric, duodenal, and colorectal epithelial neoplasms.

Patients and methods We analyzed 30 consecutive patients who were positive for the WOS, as visualized in colorectal hyperplastic polyps by ME with narrow-band imaging and 30 consecutive patients who were negative for the WOS. Biopsy specimens obtained from the polyps were immunostained with anti-adipophilin antibody to determine the correlation between the presence of the WOS and that of lipid droplets in the epithelium.

Results In all patients, the epithelial cells were histologically positive for adipophilin. However, the area of adipophilin-positive epithelial cells in the WOS-positive group was significantly larger than that in the WOS-negative group $(P<0.001)$. The density of the WOS was strongly and positively correlated with the area of adipophilin-positive cells.

Conclusions This study reveals that the WOS visualized in the superficial layers of colorectal hyperplastic polyps is produced by a dense accumulation of minute lipid droplets in the epithelia of the polyps.

\section{Introduction}

Previous reports have indicated that magnifying endoscopy (ME) of gastric, duodenal, and colorectal epithelial neoplasms (adenomas and carcinomas) reveals a white opaque substance (WOS), which obscures subepithelial microvascular patterns [1-4]. Histopathological studies have reported that the WOS

\footnotetext{
* Meeting presentations: Asian Pacific Digestive Week 2019
}

in gastric, duodenal, and colorectal epithelial neoplasms is derived from a dense accumulation of minute lipid droplets in the epithelium, which cause reflection or strong backward scattering of projected light, and are visually recognized as a white substance $[2,5,6]$. The presence of the WOS has also been reported in colorectal hyperplastic polyps [7]. However, it has been unknown whether the WOS in colorectal hyperplastic polyps is derived from a dense accumulation of lipid droplets in the epithelium, as observed in the case of gastric, duodenal, 
and colorectal epithelial neoplasms. The present study aimed to investigate the correlation between the presence of the WOS visualized in colorectal hyperplastic polyps by ME with narrow-band imaging (NBI) and lipid droplets accumulated in the epithelium.

\section{Patients and methods}

This prospective observational study was approved by the Institutional Review Board of Fukuoka University, and was registered with the University Hospital Medical Information Network (UMIN R000019211). All participating patients received an explanation of the present study and provided written informed consent.

At the Department of Endoscopy, Fukuoka University Chikushi Hospital, between February 2015 and January 2016, complete colonoscopy was performed on 2244 patients. The analyses in this study involved 30 consecutive patients with WOS-positive colorectal hyperplastic polyps and 30 consecutive patients with WOS-negative colorectal hyperplastic polyps who met the following inclusion criteria, did not meet the exclusion criteria, and were histopathologically diagnosed as having colorectal hyperplastic polyps.

The inclusion criteria were as follows: (1) patients aged 20 years or older at enrollment; (2) patients with an Eastern Cooperative Oncology Group (ECOG)-performance status of 0 or 1 ; and (3) patients who provided written informed consent to participate in the study.

The exclusion criteria were as follows: (1) lesions histopathologically diagnosed as epithelial neoplasms (adenomas/carcinomas) or non-epithelial neoplasms; (2) patients with inflammatory bowel disease (3) patients with a history of partial colectomy; (4) patients with serious underlying diseases; (5) patients with intestinal hemorrhage that posed difficulty in detailed endoscopic observation; (6) patients who might have a difficulty in participating in the study due to psychiatric conditions; and (7) other patients who were determined ineligible to participate in the study by the principal investigator or sub investigators.

\section{Endoscopic procedures and biopsy}

All endoscopic procedures were performed using an electronic endoscopy system (EVIS LUCERA ELITE; Olympus Medical Systems Co., Tokyo, Japan) with magnifying colonoscopy (CFHQ290ZI, PCF-Q260AZI; Olympus). A soft black hood attachment was mounted at the tip of the scope to obtain a consistent focal distance between the tip of the scope and a mucosal lesion at maximal magnification. Lesions were visualized using incremental movements of the tip of the endoscope to enable image focusing, with the distally attached soft black hood stabilizing the tip of the endoscope without causing mucosal injury [8]. Pretreatment for complete colonoscopy was performed as follows. The patients consumed a low-residue diet on the day before the examination, received oral sodium picosulfate (Laxoberon, Teijin Pharma, Tokyo, Japan) before bedtime, and drank $2 \mathrm{~L}$ of polyethylene glycol (MOVIPREP, EA Pharma, Tokyo, Japan) solution on the morning of the examination.
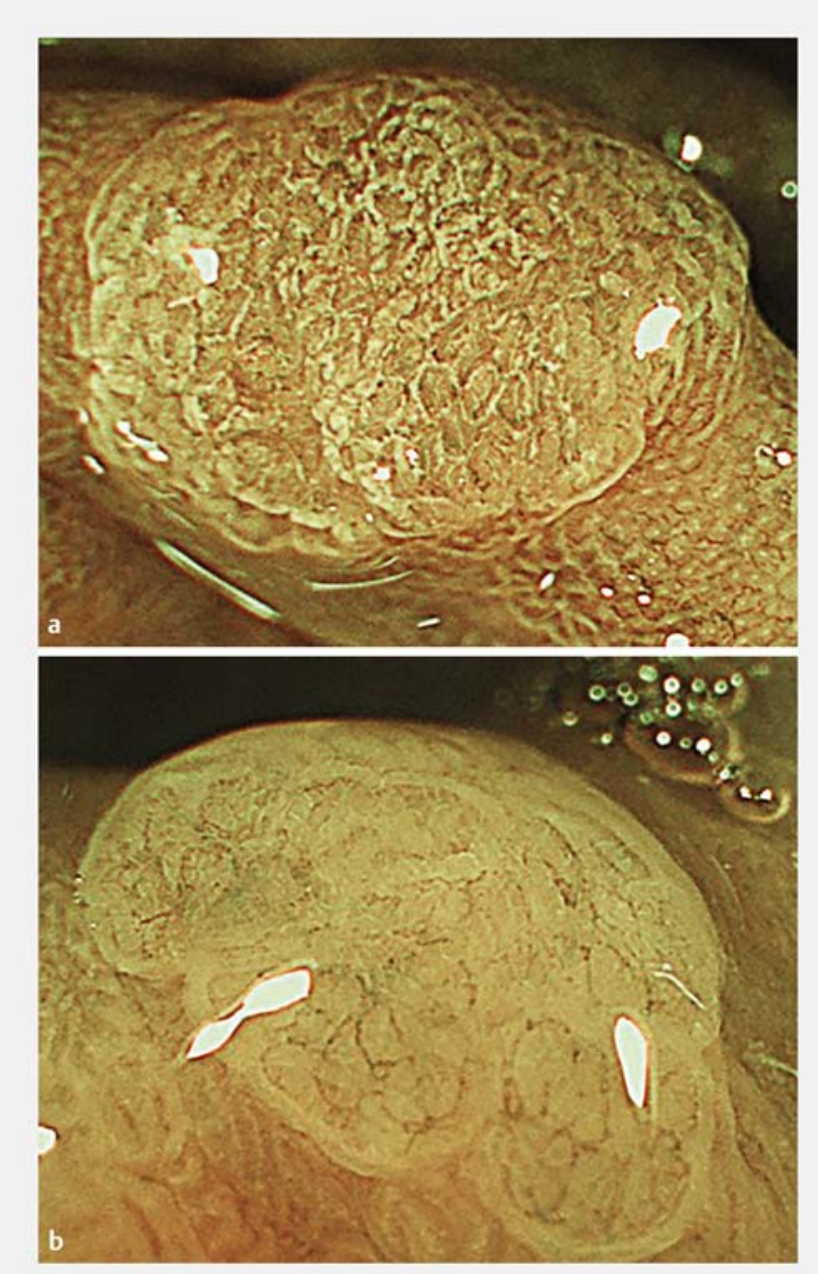

Fig. $1 \mathrm{M}-\mathrm{NBI}$ findings for colonic hyperplastic polyps. a M-NBI finding for colonic hyperplastic polyp in which WOS was present. b M-NBI finding for colonic hyperplastic polyp in which no WOS was present. M-NBI, magnifying endoscopy with narrow-band imaging

When non-ME with white light imaging revealed polyps, their surfaces were immediately irrigated and observed using nonME with NBI. When the polyps were classified as type 1 according to the NBI International Colorectal Endoscopic (NICE) classification [9], ME with NBI was performed to determine whether the WOS existed in the epithelia of the polyps. The presence or absence of the WOS was determined by ME at a maximum magnification ( $\triangleright$ Fig. 1). WOS was defined as a white substance obscuring the subepithelial microvascular pattern, which was present within the colorectal superficial epithelium. When WOS was observed in the superficial layer of a colorectal hyperplastic polyp, the lesion was determined to be positive for WOS. When no WOS was observed, the lesion was determined to be negative. Target biopsy was performed on the polyps. The WOS density was classified into grades 0 to 3 using the following WOS grading scale [10]: grade 0 , no evidence of WOS within the colorectal hyperplastic polyp; grade 1, wOS observed in up to one-third of the colorectal hyperplastic polyp; grade 2, wOS 


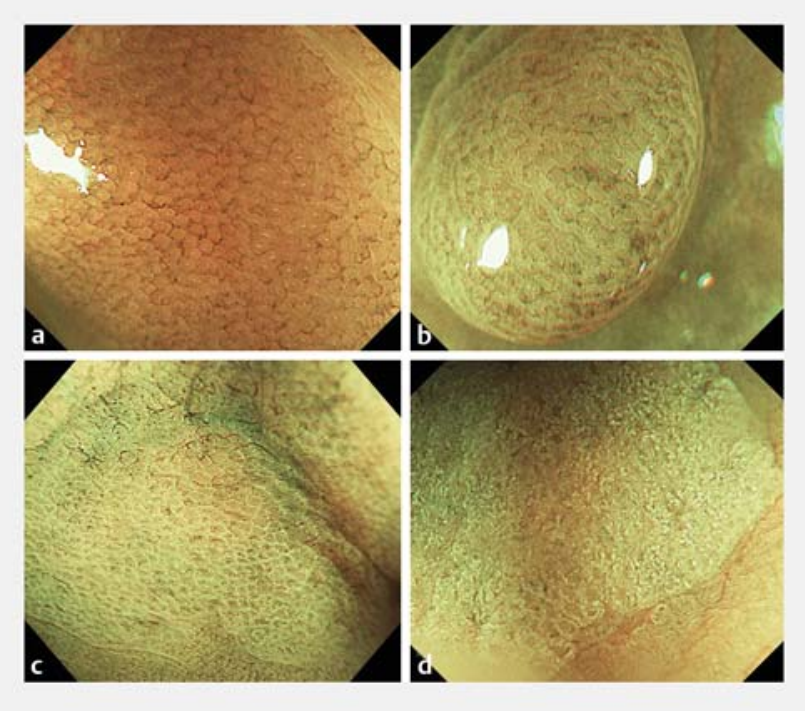

- Fig. 2 White opaque substance (WOS) grading scale. a Grade 0: no evidence of WOS within the colorectal hyperplastic polyp. b Grade 1: WOS observed in up to one-third of the colorectal hyperplastic polyp.c Grade 2: WOS observed in more than one-third and up to two-thirds of the colorectal hyperplastic polyp. $\mathbf{d}$ Grade 3: WOS observed in more than two-thirds of the colorectal hyperplastic polyp.

observed in more than one-third but up to two-thirds of the colorectal hyperplastic polyp; grade 3, wOS observed in more than two-thirds of the colorectal hyperplastic polyp ( $\triangleright$ Fig. 2). Macroscopic classification was conducted according to the updated Paris classification [11]. After formalin fixation, biopsy tissue specimens were sliced and used for hematoxylin and eo$\sin (\mathrm{HE})$ staining. and immunostaining with anti-adipophilin antibody as the primary antibody (1:50, AP125, Acris Antibodies, San Diego, California, United States) [6].

\section{Histopathologic analysis}

A pathologist (H.T.) who was blinded to the endoscopic findings made diagnoses and performed histological evaluation of adipophilin in specimens diagnosed as colorectal hyperplastic polyps. Colorectal hyperplastic polyps were histologically classified according to the World Health Organization classification [12]. First, the presence or absence of adipophilin-positive epithelial cells in the colorectal hyperplastic polyps was determined in the immunostained specimens. Next, the area that stained positive for adipophilin was quantified. The immunostained area was investigated using a light microscope (ECLIPSE 80i; Nikon, Tokyo, Japan) equipped with a digital camera (DSFi3; Nikon, Tokyo, Japan). The obtained images were transferred to a personal computer (Windows, Microsoft, Redmond, Washington, United States). The adipophilin-positive area was then measured using image integration software (NIS-Elements; Nikon, Tokyo, Japan). Specifically, we first selected 25 epithelial cells that exhibited strong positive staining for adipophilin according to a histopathological specimen biopsy (immunohistological staining with anti-adipophilin antibody as the

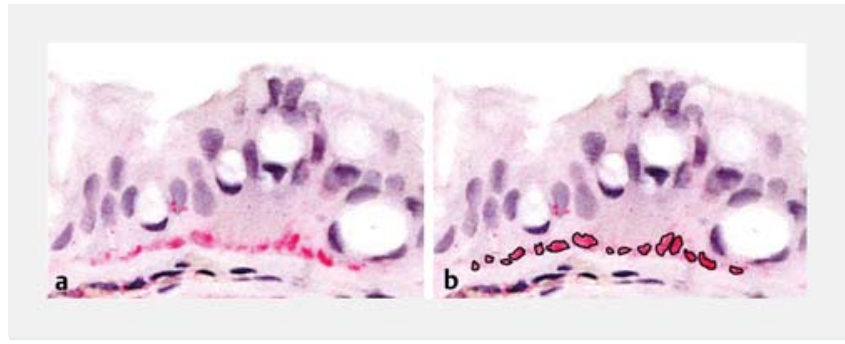

- Fig. 3 Histopathological findings from the biopsy specimens (immunohistological staining with anti-adipophilin antibody as the primary antibody). a Twenty-five adipophilin-positive epithelial cells. b Selected regions of interest in the adipophilin-positive areas. The areas enclosed with black lines are the adipophilin-positive areas found in the epithelial cells including the 25 cells.

primary antibody) ( $\triangleright$ Fig. 3a). We then used the image integration software to select regions of interest in the adipophilin-po-

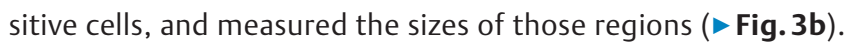
Finally, we compared the mean adipophilin-positive areas in the WOS-positive and WOS-negative colorectal hyperplastic polyps. We were therefore able to determine the correlation between the WOS grading scale and the adipophilin-positive areas.

\section{Interobserver and intraobserver agreement}

Thirty WOS-positive and 30 WOS-negative magnified endoscopic images were extracted from among the subjects for use in the testing of interobserver and intraobserver agreement. The endoscopic images were arranged in a random order. To assess interobserver agreement, two endoscopists (K.I. with 13 years of experience in performing endoscopy and A.H. with 8 years of experience) reviewed all images and determined the presence or absence of WOS in polyps in each picture. Three months later, to assess intraobserver agreement, K.I. again reviewed all images and determined the presence or absence of WOS [13].

\section{Statistical analysis}

Statistical analysis was performed using SPSS software version 21] for Windows (SPSS, Chicago, Illinois, United States). Mean and median values were compared using a Student's t-test and a Mann-Whitney $U$ test, respectively. Comparisons of the prevalence of WOS between the two groups were made using a Fisher exact test. A Spearman rank correlation coefficient was used to determine the correlation between two parameters. $P<0.05$ was considered statistically significant.

\section{Results}

Among the patients who underwent colonoscopy between February 2015 and January 2016, 149 consented to participate in this study. After the exclusion of two patients who withdrew their informed consent and 54 patients who did not have any National Institute for Health and Clinical Excellence (NICE) Type 1 lesions, there remained 93 patients with NICE Type 1 lesions, which were the target lesions. Fifty-two patients with WOS-positive colorectal hyperplastic polyps and 41 patients 
Patients enrolled in the clinical study February 2015 to January $2016(n=149)$

\begin{tabular}{|c|c|}
\hline & $\begin{array}{l}\text { Exclusion }(n=56) \\
\text { - Withdrawal of informed } \\
\text { consent }(n=2) \\
\text { - No target lesion }(n=54)\end{array}$ \\
\hline \multicolumn{2}{|l|}{$\sqrt{ }$} \\
\hline $\begin{array}{l}\text { WOS-positive lesions } \\
(n=52)\end{array}$ & $\begin{array}{l}\text { WOS-negative lesions } \\
(n=41)\end{array}$ \\
\hline $\begin{array}{l}\text { Exclusion }(n=22) \\
\text { - Hyperplastic } \\
\text { epithelium }(n=7) \\
\text { - Neoplasm }(n=1) \\
\text { - Inflammation } \\
\quad(n=14)\end{array}$ & $\begin{array}{l}\text { Exclusion }(n=11) \\
\text { - Hyperplastic } \\
\text { epithelium }(n=4) \\
\text { - Neoplasm }(n=2) \\
\text { - Inflammation } \\
(n=5)\end{array}$ \\
\hline$\downarrow$ & $\downarrow$ \\
\hline $\begin{array}{l}\text { WOS-positive colorectal } \\
\text { hyperplastic polyps }(n=30)\end{array}$ & $\begin{array}{l}\text { WOS-negative colorectal } \\
\text { hyperplastic polyps }(n=30)\end{array}$ \\
\hline
\end{tabular}

Fig. 4 Flow diagram of patient enrollment. NICE, narrow-band imaging international colorectal endoscopic; WOS, white opaque substance.

with WOS-negative colorectal hyperplastic polyps were examined in this study. After excluding seven patients with hyperplastic epithelium, one with adenoma, and 14 with inflammation (according to the histopathological diagnoses), 30 of the 52 WOS-positive patients were included in the analyses. After excluding four patients with hyperplastic epithelium, two with adenoma, and five with inflammation, 30 of the 41 WOS-negative patients were included in the analyses ( $\nabla$ Fig. 4).

- Table 1 shows the clinicopathologic characteristics of the patients with colorectal hyperplastic polyps in the WOS-positive and WOS-negative groups. No statistically significant differences were observed regarding sex, age, polyp diameter, lesion site, macroscopic morphology, or histological type.

When immunostaining with anti-adipophilin antibody was performed in the WOS-positive and WOS-negative groups, all patients were positive for adipophilin in epithelial cells ( $\bullet$ Table 2). When we carefully compared the histological findings of the immunostained specimens with the magnified endoscopic findings, we noticed the following association between WOSpositivity in the endoscopic findings and the density of the adipophilin-positive lipid droplets in the immunostained specimens. In the WOS-negative group, although epithelial cells of colorectal hyperplastic polyps contained adipophilin-positive lipid droplets, the density of the droplets tended to be lower. The WOS-positive group showed a higher density of adipophilin-positive lipid droplets in the epithelial cells of colorectal hyperplastic polyps ( $\triangleright$ Fig.5).

The adipophilin-positive area in the epithelial cells of colorectal hyperplastic polyps had a median value of $275 \mu \mathrm{m}^{2}$
- Table 1 Patient characteristics and clinicopathologic characteristics of colorectal hyperplastic polyps.

\begin{tabular}{|c|c|c|c|}
\hline & $\begin{array}{l}\text { wos-posi- } \\
\text { tive group } \\
(\mathbf{n}=\mathbf{3 0})\end{array}$ & $\begin{array}{l}\text { WOS-nega- } \\
\text { tive group } \\
(\mathrm{n}=30)\end{array}$ & $P$ value \\
\hline \multicolumn{4}{|l|}{ Sex } \\
\hline - Male/female & $18 / 12$ & $21 / 9$ & $0.589^{1}$ \\
\hline Age (mean $\pm S D)$, years & $64 \pm 10.1$ & $64 \pm 9.0$ & $0.830^{2}$ \\
\hline $\begin{array}{l}\text { Neoplasm diameter } \\
(\text { mean } \pm \text { SD), mm }\end{array}$ & $4.0 \pm 2.7$ & $3.9 \pm 2.5$ & $0.844^{2}$ \\
\hline \multicolumn{4}{|l|}{ Lesion site } \\
\hline - Colon/rectum & $9 / 21$ & $14 / 16$ & $0.288^{1}$ \\
\hline \multicolumn{4}{|l|}{ Macroscopic morphology } \\
\hline - Is/lsp & $29 / 1$ & $29 / 1$ & $1.000^{1}$ \\
\hline \multicolumn{4}{|l|}{ Histological type } \\
\hline - Microvesicular type & 23 & 19 & $0.399^{1}$ \\
\hline - Goblet cell-rich type & 4 & 11 & $0.072^{1}$ \\
\hline - Mucin-poor type & 3 & 0 & $0.237^{1}$ \\
\hline \multicolumn{4}{|c|}{$\begin{array}{l}\text { WOS, white opaque substance; SD, standard deviation; Is, sessile type; Isp, } \\
\text { semipedunculated type. } \\
{ }^{1} \text { Fisher exact test. } \\
{ }^{2} \text { Student's } t \text {-test. }\end{array}$} \\
\hline
\end{tabular}

- Table 2 Immunohistochemical expression of adipophilin according to the presence of WOS identified by ME with NBI.

\begin{tabular}{|l|l|l|l|}
\hline \multirow{2}{*}{ WOS } & \multicolumn{2}{|l|}{ Adipophilin } \\
\cline { 2 - 3 } & Positive & Negative \\
\hline \multirow{2}{*}{\begin{tabular}{l} 
Positive $(n=30)$ \\
\cline { 2 - 3 }
\end{tabular}} & $30 / 30(100 \%)$ & $0 / 30(0 \%)$ \\
\hline Negative $(n=30)$ & $30 / 30(100 \%)$ & $0 / 30(0 \%)$ \\
\hline
\end{tabular}

ME, magnifying endoscopy; NBI, narrow-band imaging;

WOS, white opaque substance. $P=1.00$, Fisher exact test.

(range $92-548 \mu \mathrm{m}^{2}$ ) in the WOS-positive group and $50 \mu \mathrm{m}^{2}$ (range $12-142 \mu \mathrm{m}^{2}$ ) in the WOS-negative group ( $\triangleright$ Fig. $\mathbf{6}$ ). The adipophilin-positive area in the epithelial cells of colorectal hyperplastic polyps was significantly larger in the WOS-positive group than in the WOS-negative group $(P<0.001)$.

The WOS grading scale was strongly and positively correlated with the adipophilin-positive area in the epithelial cells of colorectal hyperplastic polyps (Spearman rank correlation coefficient: 0.816) (ฉ Fig. 7).

The interobserver concordance rate for the assessment of WOS-positive and WOS-negative hyperplastic polyps using ME with NBI was $53 / 60$ (88.3\%), with a kappa coefficient of 0.77 , which is considered as excellent. The interobserver concordance rate of K.I. for the assessment of WOS-positive and WOS-negative hyperplastic polyps using ME with NBI was 56/ $60(93 \%)$, with a kappa coefficient of 0.86 , which is considered as excellent. 


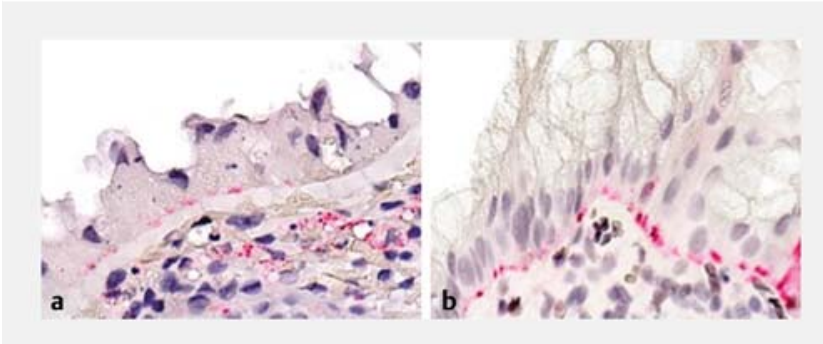

Fig. 5 Histopathological findings of biopsy specimens (immunohistological staining with anti-adipophilin antibody as the primary antibody). a White opaque substance (WOS)-negative colorectal hyperplastic polyp and $\mathbf{b}$ WOS-positive colorectal hyperplastic polyp. a Although the epithelial cells of the WOS-negative colorectal hyperplastic polyp contained adipophilin-positive lipid droplets, the density of the droplets tended to be low. $\mathbf{b}$ In epithelial cells of the WOS-positive colorectal hyperplastic polyp, the density of adipophilin-positive lipid droplets tended to be high.

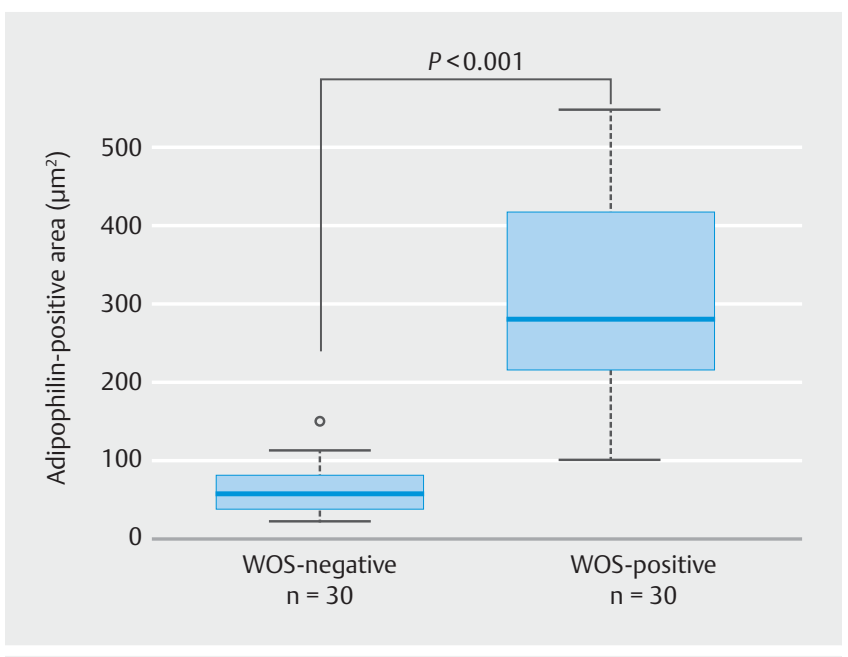

Fig. 6 Adipophilin-positive area in the epithelial cells of colorectal hyperplastic polyps in the white opaque substance (WOS)-positive and WOS-negative groups. The median adipophilin-positive area (range) in the epithelial cells of colorectal hyperplastic polyps was $275 \mu \mathrm{m}^{2}\left(92-548 \mu \mathrm{m}^{2}\right)$ in the WOS-positive group and $50 \mu \mathrm{m}^{2}$ $\left(12-142 \mu \mathrm{m}^{2}\right)$ in the WOS-negative group. The adipophilin-positive area in the epithelium of colorectal hyperplastic polyps was significantly larger in the WOS-positive group than in the WOSnegative group ( $P<0.001$, using Mann-Whitney $U$ tests).

\section{Discussion}

The present study revealed that the WOS visualized in the superficial layers of colorectal hyperplastic polyps was derived from a dense accumulation of minute lipid droplets in the epithelia of the polyps, as in the case of the WOS observed in gastric, duodenal, and colorectal neoplastic lesions [2,5,6]. Although the presence of the WOS in colorectal hyperplastic polyps has already been reported [7], it was previously unknown whether the WOS in colorectal hyperplastic polyps was derived from minute lipid droplets. To our knowledge, the present study is the first prospective observational study which de-

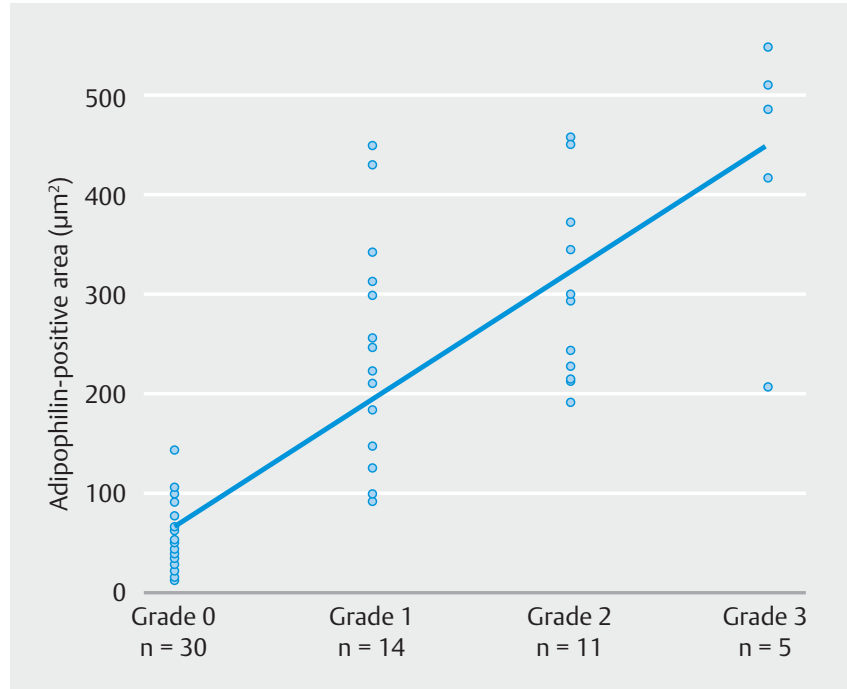

- Fig. 7 Correlation between the white opaque substance (WOS) grading scale and the adipophilin-positive area in the epithelial cells of colorectal hyperplastic polyps. The WOS grading scale was strongly and positively correlated with the adipophilin-positive area in the epithelial cells of colorectal hyperplastic polyps (Spearman rank correlation coefficient: 0.816).

monstrates that the WOS in colorectal hyperplastic polyps is derived from lipid droplets.

We performed immunostaining with anti-adipophilin antibody on specimens pathologically diagnosed as colorectal hyperplastic polyps by HE staining. We used anti-adipophilin antibody as the primary antibody for immunohistological staining because it specifically binds to the membrane proteins of lipid droplets in paraffin-embedded specimens, and has recently been used as a histological biomarker for lipid droplets [14]. Immunostaining showed that the positive rates of adipophilin expression in the epithelial cells of colorectal hyperplastic polyps were $100 \%$ (30/30) in the WOS-positive group and $100 \%$ $(30 / 30)$ in the WOS-negative groups. Thus, regardless of the presence or absence of the WOS, adipophilin-positive minute lipid droplets were observed in the epithelial cells of all colorectal hyperplastic polyps. However, when the adipophilin-positive area in the epithelium was quantified and compared between WOS-positive and WOS-negative colorectal hyperplastic polyps, it was significantly larger in the WOS-positive group than in the WOS-negative group $(P<0.001)$. The WOS grading scale was strongly and positively correlated with the adipophilin-positive area in the epithelial cells of colorectal hyperplastic polyps (Spearman's rank correlation coefficient: 0.816$)$. The mechanism by which the WOS is recognized as white coloration on ME with NBI has been discussed by Yao et al., who have suggested that when light is projected onto dense minute lipid droplets, the resultant reflection or strong backward scattering of the projected light is visually recognized as white coloration [5]. Based on that report, we hypothesized that in WOS-positive colorectal hyperplastic polyps, dense accumulation of minute lipid droplets in epithelial cells causes reflection or strong backward scattering of projected light to enable visualization of the 
droplets as the WOS. However, although minute lipid droplets exist in WOS-negative colorectal hyperplastic polyps, their density is too low to cause sufficient reflection or backward scattering of projected light to make the droplets visible as WOS. Studies on gastric and colorectal epithelial neoplasms have also reported results similar to those of the present study [5, $6]$. Thus, visualization of the WOS may require dense accumulation of minute lipid droplets in epithelial cells in both the stomach and large intestine.

The findings obtained in the present study may also be associated with the mechanism through which colorectal hyperplastic polyps appear white on endoscopy. The color of colorectal hyperplastic polyps identified using NBI has been reported to be white, whereas that of epithelial neoplasms (adenomas and carcinomas) has been reported to be brown $[9,15]$. Sano et al. have suggested that the differences in the subepithelial blood vessel diameter between neoplastic and non-neoplastic lesions are associated with the color of the lesion on NBI [15]. Sano et al. have reported that the diameter of the subepithelial blood vessels in lesions ranges from 8 to $12 \mu \mathrm{m}$ in normal colonic mucosa and colorectal hyperplastic polyps, and from 13 to $20 \mu \mathrm{m}$ in adenomas and carcinomas. These authors concluded that the mechanism by which colorectal adenomas appeared brown was due to the large diameter of the subepithelial blood vessels, and that the mechanism by which colorectal hyperplastic polyps appeared white or had no change in color was due to the small diameter of the blood vessels [15]. Hisabe et al. have reported that the WOS is common in colorectal hyperplastic polyps, occurring in $80.8 \%$ of these polyps [7]. Thus, in addition to the small diameter of subepithelial blood vessels, the reason for white coloration of colorectal hyperplastic polyps may be attributed to minute lipid droplets in the epithelium which cause reflection or backward scattering of projected light, and that reflection and backward scattering prevent a sufficient amount of projected light from reaching the subepithelial blood vessels.

Hisabe et al. compared the prevalence of the WOS between colorectal adenomas and colorectal carcinomas. They reported that the prevalence of the WOS was significantly higher in colorectal carcinomas $(66.0 \%)$ than in colorectal adenomas $(31.8 \%$; $P<0.001$ ) [4]. Kawasaki et al. have reported that colorectal adenomas are morphologically characterized by regular WOS on examination using ME, whereas colorectal carcinomas are characterized by irregular WOS [16]. They suggested that WOS might be useful for differentiation between carcinomas and non-carcinomas. However, because the WOS exists in both epithelial neoplasms and non-neoplasms, it seems impossible to make any differential diagnosis based on only the presence of the WOS. The diagnostic performance of endoscopy for colorectal hyperplastic polyps, colorectal adenomas, and colorectal carcinomas may be improved by performing ME on all these lesions to reveal the morphological and other characteristics of the WOS, as well as the presence of the WOS.

One limitation of the present study is that the biopsy specimens were too small to allow for successful measurement of the diameter of the subepithelial blood vessels. Consequently, we were unable to examine the association between the endoscopic findings and the diameter of the subepithelial blood ves- sels. Further studies are needed to investigate the association between the findings of ME with NBI and histological features such as the lipid droplets in the epithelium and the diameter and density of subepithelial blood vessels. Despite the prospective study design, the present study is a single-center study with a small sample size.

In the future, multicenter prospective studies with a larger sample size for epithelial neoplastic and non-neoplastic lesions of the large intestine are necessary to investigate how the WOS and other characteristic endoscopic findings are associated with histological features, such as the minute lipid droplets in the epithelium and subepithelial blood vessels. Based on such studies, establishment of comprehensive diagnostic criteria for colorectal epithelial lesions can be achieved.

\section{Conclusions}

In conclusion, this study demonstrated that the WOS visualized in the superficial layers of colorectal hyperplastic polyps by ME is derived from the dense accumulation of minute lipid droplets in the epithelia of the polyps.

\section{Competing interests}

The authors declare that they have no conflict of interest.

\section{References}

[1] Yao K, Iwashita A, Tanabe H et al. White opaque substance within superficial elevated gastric neoplasia as visualized by magnification endoscopy with narrow- band imaging: a new optical sign for differentiating between adenoma and carcinoma. Gastrointest Endosc 2008; 68: 574-580

[2] Tanaka M, Usuda K, OOkura Y et al. Significance of magnifying endoscopy in diagnosis of duodenal elevated lesions [in Japanese with English abstract]. Stomach and Intestine 2003; 38: 1709-1720

[3] Tsuji S, Doyama H, Tshuji K et al. Magnifying endoscopy with narrow band imaging for superficial non-ampullary duodenal epithelial tumors before biopsy [in Japanese with English abstract]. Stomach and Intestine 2016; 68: 574-580

[4] Hisabe T, Yao K, Imamura K et al. White opaque substance visualized using magnifying endoscopy with narrow-band imaging in colorectal epithelial neoplasms. Dig Dis Sci 2014; 59: 2544-2549

[5] Yao K, Iwashita A, Nambu M et al. Nature of white opaque substance in gastric epithelial neoplasia as visualized by magnifying endoscopy with narrow-band imaging. Dig Endosc 2012; 24: 419-425

[6] Imamura K, Yao K, Hisabe T et al. The nature of the white opaque substance within colorectal neoplastic epithelium as visualized by magnifying endoscopy with narrow-band imaging. Endosc Int Open 2016; 04: E1151-E1157

[7] Hisabe T, Yao K, Imamura K et al. Novel endoscopic findings as visualized by magnifying endoscopy with narrow-band imaging: white opaque substance is present in colorectal hyperplastic polyps. Digestion 2016; 93: 127-131

[8] Yao K, Anagnostopoulos GK, Ragunath K. Magnifying endoscopy for diagnosing and delineating early gastric cancer. Endoscopy 2009; 41: 462-467 
[9] Hewett DG, Kaltenbach T, Sano Y et al. Validation of a simple classification system for endoscopic diagnosis of small colorectal polyps using narrow-band imaging. Gastroenterology 2012; 143: 599-607

[10] Ohtsu K, Yao K, Matsunaga $\mathrm{M}$ et al. Lipid is absorbed in the stomach by epithelial neoplasms (adenomas and early cancers): a novel functional endoscopy technique. Endosc Int Open 2015; 03: E318-E322

[11] Endoscopic Classification Review Group. Update on the Paris classification of superficial neoplastic lesions in digestive tract. Endoscopy 2005; 37: 570-578

[12] Snover DC, Ahnen DJ, Burt RW et al. Serrated polyps of the colon and rectum and serreted polyposis.Bosman FT, Carneriro F, Hruban RH et al. World Health Organization Classification of Tumours of the Digestive System. Lyon: IARC Press; 2010: 160-165
[13] Landis JR, Koch GG. The measurement of observer agreement for categorical data. Biometrics 1977; 33: 159-174

[14] Heid HW, Moll R, Scchetlick I et al. Adipophilin is a specific marker of lipid accumulation in diverse cell types and disease. Cell Tissue Res 1998; 294: 309-321

[15] Sano Y, Horimatsu T, Fu KI et al. Magnifying observation of microvascular architecture of colorectal lesions using a narrow-band imaging system. Dig Endosc 2006; 18: 44-51

[16] Kawasaki K, Kurahara K, Yanai S et al. Significance of a white opaque substance under magnifying narrow-band imaging colonoscopy for the diagnosis of colorectal epithelial neoplasms. Gastrointest Endosc 2015; 82: 1097-1104

\section{CORRECTION}

Akikazu Hirano, Kenshi Yao, Hiroshi Ishihara et al. Nature of a white opaque substance visualized by magnifying endoscopy in colorectal hyperplastic polyps Endoscopy International Open 2021; 09: E1077-E1083. DOI: 10.1055/a-1452-9669

In the above mentioned article the name of the author Kenshi Yao was switched. Correct is: Kenshi Yao. 